Chirurg 2019 90 (Suppl 2):S28 https://doi.org/10.1007/s00104-019-0836-1 Online publiziert: 13. Februar 2019

(c) Springer Medizin Verlag GmbH, ein Teil von Springer Nature 2019

\section{Originalpublikation}

Schauer PR, Bhatt DL, Kirwan JP et al STAMPEDE Investigators (2017) Bariatric surgery versus medical therapy for diabetes 5-year outcomes. NEJM 376(7):641-651

Hintergrund und Fragestellung. In mehreren prospektiven Beobachtungsund auch randomisierten Studien konnte eindeutig ein positiver Effekt der bariatrischen Chirurgie auf den BodyMass-Index (BMI) und Diabetes mellitus nachgewiesen werden. Dennoch ist in Deutschland zur chirurgischen Therapie der morbiden Adipositas eine Beantragung zur Kostenübernahme durch die Krankenkasse für jeden Patienten erforderlich, eine generelle Listung dieses Eingriffs in den Leistungskatalog der Krankenkasse wie von der Deutschen Gesellschaft für Allgemein- und Viszeralchirurgie (DGAV) gefordert wird gegenwärtig abgelehnt. In diesem $\mathrm{Zu}$ sammenhang ist eine aktuelle Studie eines amerikanischen Zentrums wichtig, in welcher die Langzeitergebnisse der bariatrischen Chirurgie auf den Zuckerstoffwechsel und den BMI mit der alleinigen medikamentösen Therapie verglichen werden.

Methoden. In einem monozentrischen prospektiv-randomisierten dreiarmigen Studiendesign wurden insgesamt $134 \mathrm{~Pa}$ tienten vergleichend nach 5 Jahren analysiert (38 Patienten mit alleiniger medikamentöser Therapie, 49 Patienten mit

Dieser Beitrag wurde erstpubliziert in Der Chirurg (2017) 88:449-450. https://doi.org/10. 1007/s00104-017-0426-z

W. Schröder · C. Bruns

Klinik für Allgemein-, Viszeral- und Tumorchirurgie, Universitätsklinik Köln, Köln, Deutschland

\title{
Bariatrische Chirurgie versus medikamentöse Therapie in der Behandlung der Adipositas
}

Magenbypass, 47 Patienten mit "gastric sleeve"). Der durchschnittliche BMI dieser Studiengruppe lag bei $37 \pm 3,5$, der durchschnittliche HbA1c („glycated hemiglobin“) bei 9,2 \pm 1,5\%. Primärer Zielparameter war die Reduktion des HbAlc unter $6 \%$ mit oder ohne Diabetesmedikation. Sekundärer Endpunkt waren die Reduktion des BMI sowie Parameter der Lebensqualität. Auch die chirurgischen Patienten wurden postoperativ einer intensivierten medizinischen Überwachung und Therapie unterzogen.

Ergebnisse. Nach einer Beobachtungszeit von 5 Jahren wurde das primäre Zielkriterium eines $\mathrm{HbA} 1 \mathrm{c}<6 \%$ in der medikamentös therapierten Gruppe von nur $5 \%$ der Patienten erreicht und war damit signifikant geringer als in Gruppe mit Magenbypass $(29 \%$, , unadjusted“ $p=$ $0,01)$ und der Gruppe mit "gastric sleeve“ (23\%, ,unadjusted“ $p=0,03)$. Eine ähnliche Beobachtung wurde für den BMIVerlauf gemacht. Bezogen auf das Ausgangsgewicht kam es bei Patienten mit Magenbypass zu einer signifikanten Gewichtsreduktion von $23 \%$, bei Patienten mit "gastric sleeve“ von $19 \%$ und bei Patienten mit alleiniger medikamentöser Therapie lediglich zu einer Gewichtsreduktion von $5 \%$. Auch die ebenfalls analysierten Parameter der Lebensqualität waren bei den chirurgisch therapierten Patienten signifikant besser.

Fazit. Die vorliegende Studie lässt keinen Zweifel daran, dass die beiden bariatrischen Operationen, der Magenbypass und der "gastric sleeve“ der alleinigen medikamentösen Therapie in allen gemessenen Endpunkten überlegen sind, dieses insbesondere auch im Langzeitverlauf. Aufgrund der niedrigen Fallzahl konnte keine statistische Aussage zugunsten eines der beiden eingesetzten Operationsverfahren gemacht werden, auch wenn die vorliegenden Ergebnisse tendenziell den Magenbypass als bariatrische Operation favorisieren. Die Langzeitergebnisse dieser Studie mit hohem Evidenzgrad sind ein weiteres Argument dafür, bariatrische Operationen als Standardtherapie der Adipositas und ihrer Folgeerkrankungen nicht nur in den Leitlinien $\mathrm{zu}$ verankern, sondern auch die Bereitstellung finanzieller Mittel als Regelleistung der Krankenkassen einzufordern.

\section{Korrespondenzadresse}

Prof. Dr. W. Schröder, FACS, FEBS

Klinik für Allgemein-, Viszeral- und Tumorchirurgie, Universitätsklinik Köln Kerpener Str. 62, 50937 Köln, Deutschland wolfgang.schroeder@uni-koeln.de

Interessenkonflikt. W. Schröder und C. Bruns geben an, dass kein Interessenkonflikt besteht. 